\title{
La dynamique des possibles. Incertitude et durabilité dans les pratiques de transformation urbaine
}

\section{Margot Pellegrino}

\section{CpenEdition}

Journals

Édition électronique

URL : http://journals.openedition.org/cdg/2231

DOI : $10.4000 /$ cdg.2231

ISSN : 2107-7266

Éditeur

UMR 245 - CESSMA

Référence électronique

Margot Pellegrino, « La dynamique des possibles. Incertitude et durabilité dans les pratiques de transformation urbaine », Carnets de géographes [En ligne], 1 | 2010, mis en ligne le 01 octobre 2010 consulté le 24 septembre 2020. URL : http://journals.openedition.org/cdg/2231 ; DOI : https://doi.org/ $10.4000 /$ cdg. 2231

Ce document a été généré automatiquement le 24 septembre 2020.

\section{(†) $\Theta$}

La revue Carnets de géographes est mise à disposition selon les termes de la Licence Creative Commons Attribution - Pas d'Utilisation Commerciale - Pas de Modification 4.0 International. 


\title{
La dynamique des possibles. Incertitude et durabilité dans les pratiques de transformation urbaine
}

\author{
Margot Pellegrino
}

\section{Présentation de la problématique}

1 Objectif de la thèse : analyser le thème de l'incertitude dans l'aménagement urbain ; étudier la prise en considération de l'incertitude dans les processus de planification.

2 La recherche met en évidence le rôle de l'incertitude dans la transformation de la ville, comparée à la durabilité. Cet aspect - qui devrait être objet d'autres études plus approfondies - s'applique également dans les cas hors de l'Europe. Le problème abordé dans la thèse - celui de « l'incertitude positive » dans le processus du projet - interroge la nécessité de définir des outils capables d'identifier l'aléa des processus. La possibilité d'identifier une "incertitude positive» dans les pratiques du projet urbain est construit à partir de critères d'édification axés sur la notion de durabilité.

3 La crise de l'industrie européenne des années soixante-dix et quatre-vingt du siècle dernier a conduit au développement d'immenses zones urbaines désaffectées. Cet événement ne peut être clairement conféré aux seules compétences et intérêts propres de la planification et de l'architecture: la complexité de l'effort économique et l'énergie qui doit être dépensée dans le processus sont immenses et elles exigent donc que d'autres acteurs soient impliqués dans l'évaluation de la gestion et la planification : des économistes, des philosophes, des sociologues et des écrivains sont mobilisés dans l'étude. Par ailleurs, la thèse met l'accent sur la façon dont le facteur d'incertitude (et la crise du système) peut être considéré comme endogène dans la société européenne et occidentale. L'incertitude est donc un phénomène intrinsèque de la société qui, s'il 
émerge et est reconnu, conduit à la détermination de réponses et de stratégies opérationnelles.

4 Le principe est de mettre en évidence la façon dont la recherche opérationnelle essaie de considérer comme positive la «catégorie de l'incertitude». Elle devient en fait un moteur événementiel, élément décisif pour la détermination des processus historiques de changement social et structural sur le territoire. L'incertitude modifie les règles de la planification et de l'utilisation des outils qui deviennent multi-textuels et souples, flous, avec une approche multi-scalaire. La thèse poursuit en analysant des cas d'étude, choisis parce qu'ils abordent la prise en considération de l'incertitude dans le processus de planification et de gestion de projets. Parmi les points faibles on note une approche souple et dynamique, qui ne garantit pas la cohérence mutuelle et générant une absence de forme et non de structure.

5 Les conclusions et les réflexions portent essentiellement sur une comparaison méthodologique entre l'incertitude et la durabilité, avec la prise de conscience que, au contraire de l'incertitude, le développement durable met l'accent sur la qualité et sur son contrôle et rend plus concrète l'interdépendance des acteurs dans le processus.

\section{Méthode de recherche}

La volonté est celle de traiter les thématiques de recherche avec une approche interdisciplinaire et multi échelle, basée sur les notions propres de l'innovation, en faisant référence à une méthode d'évaluation comparable au LCA (Life Cycle Assessment) dans le but d'évaluer le projet en tant que processus. Les éléments sont mis en évidence avec des parcours non seulement cycliques ou fluides, continus ou divergents.

\section{Aire géographique traitée}

7 Le choix des cas d'études se fait en deux catégories:

- prise de conscience générale de la notion d'incertitude (projets 'précurseurs': Lille, Strasbourg, Malmö, Saint Denis, Milano, Venezia)

- outils et réalisations architecturales : projets de Nantes (Ile de Nantes) et Bordeaux (Cœur Bastide).

Fiche informative

Discipline

Architecture / Géographie

Directeurs

S. Gron, A Magnaghi (École Polytechnique de Turin) ; C. Vallat (Paris Ouest Nanterre La Défense)

Université

École doctorale « Storia e valorizzazione del patrimonio architettonico, urbanistico e ambientale » de l'école Polytechnique de Turin / École doctorale « Milieux, cultures sociétés du passé et du présent » de l'université Paris Ouest Nanterre La Défense 
Membres du jury de thèse, soutenue en mai 2010

- S. Gron (professeur à l'Université polytechnique de Turin)

- C. Vallat (Professeur à l'université Paris Ouest Nanterre La Défense)

- A. Milan (professeur à l'Université de Bologne)

- D. Rivière (Professeur à l'Université Denis-Diderot Paris7)

- F. Maccaglia (Maître de conférences è l'université de Tours).

Situation professionnelle actuelle

Post-doc à l'Université de Jadavpur, Kolkata, Inde.

Contact de l'auteur

margot233[at]tiscali.it

INDEX

Thèmes : Carnets de soutenances 